\title{
Serum heparan sulfate and chondroitin sulfate concentrations in patients with newly diagnosed exfoliative glaucoma
}

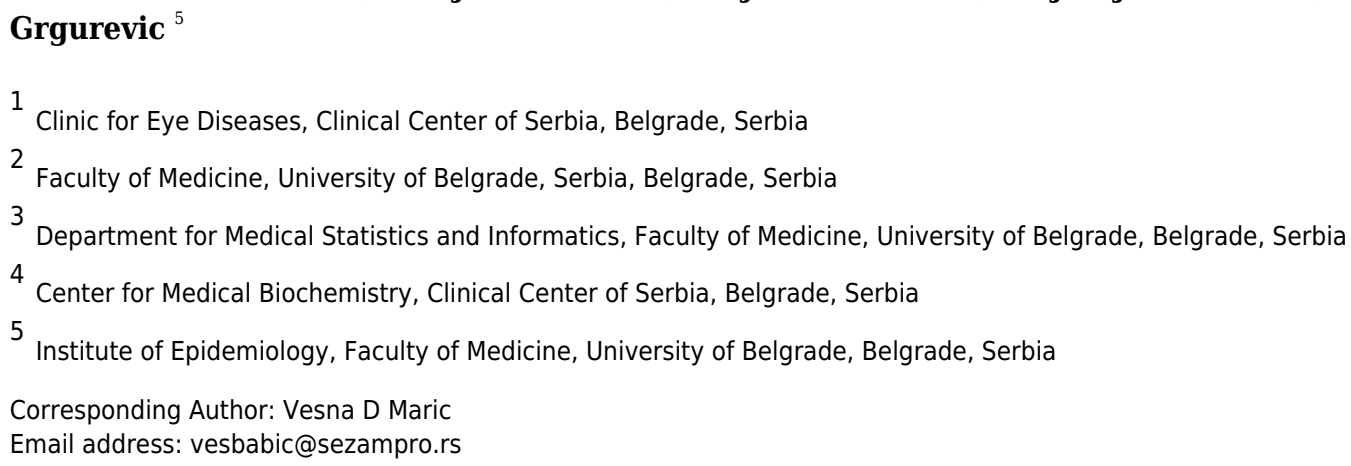

Background. Exfoliative glaucoma (XFG) is typically classified as a high-pressure type of secondary open-angle glaucoma that develops as a consequence of exfoliation syndrome (XFS). XFS is an age-related, generalized disorder of the extracellular matrix characterized by production and progressive accumulation of a fibrillar exfoliation material in intra- and extraocular tissues. Exfoliation material represents complex glycoprotein/proteoglycan structure composed of a protein core surrounded by glycosaminoglycans such as heparan sulfate (HS) and chondroitin sulfate (CS). The purpose of the present study was to investigate HS and CS concentrations in serum samples of patients with newly diagnosed XFG and compare the obtained values with those pertaining to newly diagnosed primary open-angle glaucoma (POAG), normal controls (NC) and subjects with XFS. Methods. This case control study involved 165 subjects, including patients with newly diagnosed XFG, patients with newly diagnosed POAG, subjects with XFS, and age- and sex-matched NC. The study was conducted at the Glaucoma Department of Clinic for Eye Diseases, Clinical Centre of Serbia, as the referral center for glaucoma in Serbia. Results. The mean age in the XFG, POAG, XFS and NC groups was 73.3 $\pm 9.0,66.3 \pm 7.8,75.5 \pm 7.0$ and $73.5 \pm 9.5$ years, respectively, XFG vs. POAG, $p<0.001$. Mean serum HS concentrations in the XFG, POAG, NC and XFS groups were 3,189.0 $\pm 1,473.8 \mathrm{ng} / \mathrm{mL}, 2,091.5 \pm 940.9 \mathrm{ng} / \mathrm{mL}, 2,543.1$ $\pm 1,397.3 \mathrm{ng} / \mathrm{mL}$ and 2,658.2 $\pm 1,426.8 \mathrm{ng} / \mathrm{mL}$ respectively, XFG vs. POAG, $p=0.001$ and XFG vs. NC, $p=0.032$. Mean serum CS concentrations in the XFG, POAG, NC and XFS group were $43.9 \pm 20.7 \mathrm{ng} / \mathrm{mL}, 38.5 \pm 22.0 \mathrm{ng} / \mathrm{mL}, 35.8 \pm 16.4 \mathrm{ng} / \mathrm{mL}$ and $43.3 \pm 21.8$ $\mathrm{ng} / \mathrm{mL}$, respectively, XFG vs. NC, $\mathrm{p}=0.041$. Conclusions. Our findings revealed greater HS and CS concentrations in XFG patients and XFS subjects compared to those without exfoliation material. Implications of HS and CS in the pathophysiology of exfoliation 
syndrome and glaucoma should be studied further. Serum is easily accessible and should thus be explored as rich sources of potential biomarkers. Further research should aim to identify XFG biomarkers that could be utilized in routine blood analysis tests, aiding in timely disease diagnosis. 
1 Serum heparan sulfate and chondroitin sulfate concentrations in patients with newly

2 diagnosed exfoliative glaucoma

3

4

5

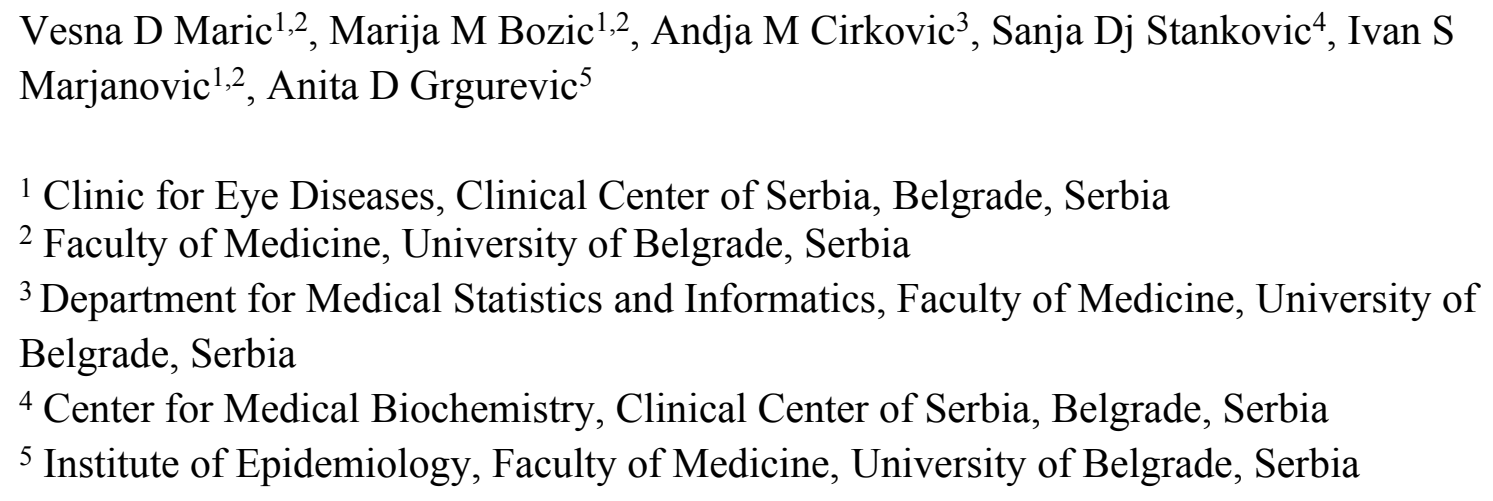

\section{Abstract}

Background. Exfoliative glaucoma (XFG) is typically classified as a high-pressure type of secondary open-angle glaucoma that develops as a consequence of exfoliation syndrome (XFS). XFS is an age-related, generalized disorder of the extracellular matrix characterized by production and progressive accumulation of a fibrillar exfoliation material in intra- and extraocular tissues. Exfoliation material represents complex glycoprotein/proteoglycan structure composed of a protein core surrounded by glycosaminoglycans such as heparan sulfate (HS) and chondroitin sulfate (CS). The purpose of the present study was to investigate HS and CS concentrations in serum samples of patients with newly diagnosed XFG and compare the obtained values with those pertaining to newly diagnosed primary open-angle glaucoma (POAG), normal controls (NC) and subjects with XFS.

Methods. This case control study involved 165 subjects, including patients with newly diagnosed XFG, patients with newly diagnosed POAG, subjects with XFS, and age- and sexmatched NC. The study was conducted at the Glaucoma Department of Clinic for Eye Diseases, Clinical Centre of Serbia, as the referral center for glaucoma in Serbia.

Results. The mean age in the XFG, POAG, XFS and NC groups was 73.3 $\pm 9.0,66.3 \pm 7.8,75.5$ \pm 7.0 and $73.5 \pm 9.5$ years, respectively, XFG vs. POAG, $p<0.001$. Mean serum HS concentrations in the XFG, POAG, NC and XFS groups were 3,189.0 $\pm 1,473.8 \mathrm{ng} / \mathrm{mL}, 2,091.5$ $\pm 940.9 \mathrm{ng} / \mathrm{mL}, 2,543.1 \pm 1,397.3 \mathrm{ng} / \mathrm{mL}$ and 2,658.2 $\pm 1,426.8 \mathrm{ng} / \mathrm{mL}$ respectively, XFG vs. POAG, $p=0.001$ and XFG vs. NC, $p=0.032$. Mean serum CS concentrations in the XFG, POAG, NC and XFS group were $43.9 \pm 20.7 \mathrm{ng} / \mathrm{mL}, 38.5 \pm 22.0 \mathrm{ng} / \mathrm{mL}, 35.8 \pm 16.4 \mathrm{ng} / \mathrm{mL}$ and $43.3 \pm 21.8 \mathrm{ng} / \mathrm{mL}$, respectively, XFG vs. $\mathrm{NC}, \mathrm{p}=0.041$. 
43 Conclusions. Our findings revealed greater HS and CS concentrations in XFG patients and XFS 44 subjects compared to those without exfoliation material. Implications of HS and CS in the 45 pathophysiology of exfoliation syndrome and glaucoma should be studied further. Serum is 46

\section{Introduction}

Glaucoma is a term describing a group of ocular disorders with multi-factorial etiology and it is a form of optic neuropathy related to elevated intraocular pressure as the main risk factor (Weinreb \& Aung \& Medeiros, 2014).

It is the second leading cause of blindness worldwide (Quigley, 2006), with primary open-angle glaucoma (POAG) and exfoliative glaucoma (XFG) as its most prevalent types in developed countries (Prum et al., 2016; Hollo \& Katsanos \& Konstas, 2015).

Exfoliation syndrome (XFS) is an age-related, generalized disorder of the extracellular matrix (ECM) characterized by production and progressive accumulation of a fibrillar exfoliation material (XFM) deposits in tissues throughout the anterior segment, as well as in connective tissues comprising various visceral organs (Ritch \& Schlötzer-Schrehardt \& Konstas, 2003; Ritch \& Schlötzer-Schrehardt, 2001). The most widely recognized disease manifestation is XFG, while glaucoma develops in only about $30 \%$ of XFS eyes in patients' lifetime (Ritch, 2015).

Exfoliative glaucoma is typically classified as a high-pressure type of secondary open-angle glaucoma (Puska, 2015). Compared with POAG, more adverse clinical progression and poorer prognosis is usually associated with XFG (Ritch \& Schlötzer-Schrehardt, 2001).

According to the findings yielded by immunohistochemical and biochemical studies, exfoliation material (XFM) represents a highly glycosylated, cross-linked, and enzymatically resistant glycoprotein/proteoglycan complex (Zenkel \& Schlötzer-Schrehardt, 2014; Schlötzer-Schrehardt \& Naumann, 2015). Proteoglycans consist of a core protein to which glycosaminoglycan (GAG) side chains are attached. GAGs are an important component of the XFM. They are linear polysaccharides comprised of glucosamine-/ galactosamine-containing repeating disaccharides (Yue, 2014). There are four GAG groups: hyaluronic acid, keratan sulfate, chondroitin/dermatan sulfate, and heparan sulfate, including heparin.

In both XFS and XFG, the progressively accumulating XFM successively detaches, destroys, and replaces the normal extracellular matrix, e.g., the basement membranes of the cells, and ultimately results in degeneration of the cells involved (Schlötzer-Schrehardt \& Naumann, 2006; Schlötzer-Schrehardt, 2012). Thus, overproduction and abnormal metabolism of glycosaminoglycans has been suggested as one of the key changes in exfoliation syndrome (Baba, 1983; Schlötzer-Schrehardt \& Dorfler \& Naumann, 1992). As noted above, heparan sulfate (HS) and chondroitin sulfate (CS) are two major GAG types (Lu et al., 2010; Li et al., 2017). Therefore, ability to quantify changes in blood GAG structures will advance the understanding and diagnosis of human diseases ( $\mathrm{Lu}$ et al., 2010).

The purpose of the present study was to investigate heparan sulfate and chondroitin sulfate concentrations in serum samples of patients with newly diagnosed untreated XFG and compare the obtained values with those pertaining to newly diagnosed untreated primary open-angle glaucoma (POAG), normal controls and subjects with XFS. The second goal was to investigate 
88 the relationship between the HS and CS serum concentrations and clinical parameters in XFG, 89 POAG and XFS groups.

90

91

92

93

94

95

96

97

98

99

100

101

102

103

104

105

106

107

108

109

110

111

112

113

114

115

116

117

118

119

120

121

122

123

124

125

126

127

128

129

130

131

132

133

\section{Materials \& Methods Study population}

This case control study involved 165 consecutive patients that were seen between June 2016 and December 2017 at the Glaucoma Department of Clinic for Eye Diseases, Clinical Centre of Serbia, as the referral center for glaucoma in Serbia. The sample comprised of patients with newly diagnosed XFG and age- and sex-matched normal controls, along with patients with newly diagnosed POAG and subjects with XFS. Subjects were classified as having POAG if they presented with the typical glaucomatous optic disc (neural rim thinning or notching, saucerization, thin nasal rim or total cupping) and/or glaucoma visual field changes, in the presence of an IOP $\geq 22 \mathrm{~mm} \mathrm{Hg}$ without medication, and a gonioscopy finding of a wide and open anterior chamber angle. XFG was diagnosed based on typical glaucomatous optic disc and/or glaucoma visual field changes in the presence of an IOP $\geq 22 \mathrm{~mm} \mathrm{Hg}$ without medication, with presence of exfoliation on the pupil edge and/or the anterior lens capsule after mydriasis by biomicroscopic evaluation in either or both eyes. Finally, XFS diagnosis was established by visualization of XFM on the pupillary margin and/or on the anterior lens surface after pupillary dilation, along with an IOP $<22 \mathrm{~mm} \mathrm{Hg}$, in the absence of glaucomatous optic nerve damage and visual field changes. The patient was classified as having XFS if XFM was present in either or both eyes. Normal control subjects had no evidence of XFS or glaucoma based on clinical examination.

Exclusion criteria were: (1) use of anti-glaucoma medications; (2) use of topical/systemic steroids; (3) previous intraocular surgery; (4) history of ocular trauma, uveitis, corneal scars, lens-induced glaucoma, proliferative diabetic retinopathy and any other ocular pathology that could have led to secondary glaucoma; (5) diseases that could influence the HS and CS levels, such as any type of cancer or rheumatoid arthritis and late-stage osteoarthritis (Li et al., 2017; Pothacharoen et al., 2006). Anti-glaucoma medications were not used by 244 subjects, 6 of whom had intraocular surgery and 15 used topical and systemic steroids in the six months prior to joining the study. In addition, 35 of the remaining 223 subjects were excluded due to history of ocular trauma, uveitis, corneal scars, lens-induced glaucoma and proliferative diabetic retinopathy. Further 23 subjects were excluded from the study due to comorbidity that could influence the HS and CS levels: cancer (10), rheumatoid arthritis and late-stage osteoarthritis diseases (13). Thus, the final sample comprised of 165 subjects.

All subjects that met the study inclusion criteria received a detailed explanation of the study purpose and the nature of their involvement, and those that agreed to take part in the investigation signed an informed consent form, in accordance with the principles embodied in the Declaration of Helsinki. The study as a part of the doctoral dissertation was reviewed and approved by the Ethics Committee of the Faculty of Medicine, University of Belgrade, record number 29/III-3.

\section{Collecting data}

Participants' demographic and comorbidity characteristics were obtained via interviews and by reviewing medical documentation. While age and gender were the only demographic data of interest, comorbidities included presence of systemic diseases, such as diabetes mellitus (DM), 
134 systemic hypertension, history of myocardial stroke, history of coronary artery bypass or 135 vascular surgery, history of abdominal aortic aneurysm, arrhythmia, and history of acute 136 cerebrovascular disease.

137

\section{Eye examinations}

139 Ocular examination in all patients was performed by one ophthalmologist (VM) and included 140 visual acuity, slit-lamp biomicroscopy, gonioscopy (using Goldmann two-mirror indirect

141

142

143

144

145

146

147

148

149

150

151

152

153

154

155

156

157

158

159

160

161

162

163

164

165

166

167

168

169

170

171

172

173

174

175

176

177

178 gonioscope), IOP measurement (using Goldmann applanation tonometry) and dilated fundus examination (using Volk Superfield $+90 \mathrm{D}$ lens). The mean IOP based on three readings in each eye was adopted as the pressure for that eye. A visual field test was performed using the Threshold C 24-2 Swedish Interactive Testing Algorithm (SITA) standard program with Humphrey Visual Field Analyzer II (Carl Zeiss, Germany). Visual acuity (VA) was measured by Snellen chart at $6 \mathrm{~m}$ distance and was converted to decimal notation, whereby the best-corrected visual acuity (BCVA) was recorded. If the patients were unable to read any letters displayed on the chart, their ability to count fingers was used as the VA. The next lower level of vision would be indicated by the ability to perceive light (denoted as "LP" or "light perception"). Complete blindness was diagnosed if no light perception ("NLP") was determined by clinical examination. If the anterior chamber angle was open, participants had their pupils dilated by administering dilation drops containing 5\% phenylephrine and $1 \%$ tropicamide. Prior to pupil dilation, a detailed high-magnification slit-lamp assessment of the pupil margin was carried out. After pupil dilation, the anterior lens surface in each eye was scanned, looking specifically for signs of XFM. If the angle was potentially occludable, the lens and the fundus evaluations were performed without dilation, and the participants were referred for a laser peripheral iridotomy. In these cases, dilated lens and fundus examinations were performed upon iridotomy completion. Indices of glaucoma severity were expressed numerically as cup to disc ratio $(\mathrm{C} / \mathrm{D})$, allowing the vertical $\mathrm{C} / \mathrm{D}(\mathrm{vC} / \mathrm{D})$ to be reported, along with the staging of visual field defects using Hodapp Classification, and separately visual field mean deviation (MD) and pattern standard deviation (PSD).

\section{Blood Sampling}

After eye examinations, blood samples were collected from each subject. Samples were collected into a serum separator tube. Blood was coagulated at room temperature for two hours and was subsequently centrifuged at approximately $1000 \times \mathrm{g}$ for 15 minutes. Serum was separated into aliquots and was stored at $-70{ }^{\circ} \mathrm{C}$ until required for analyses.

\section{Assay}

Serum human heparan sulfate concentration was measured using commercial ELISA Kit (Cusabio, USA). This assay employs the competitive inhibition enzyme immunoassay technique and has a $20-8,000 \mathrm{ng} / \mathrm{mL}$ measurement range, with $8 \mathrm{ng} / \mathrm{mL}$ detection limit. When using this ELISA Kit, intra-assay and inter-assay precision $<6 \%$ and $<11 \%$ is achieved, respectively. In this work, antibody specific to HS was pre-coated onto a microplate and standards and samples were pipetted into the wells with a Horseradish Perxidase (HRP) conjugated HS. A competitive inhibition reaction was launched between HS (standards or samples) and HRT-conjugated HS with the pre-coated antibody specific for HS. Following a wash to remove any unbound reagent, a substrate solution was added to the wells, whereby the developed color is inversely 
179 proportional to the amount of HS in the sample. Thus, when color development is terminated, 180 color intensity is measured and is converted to the corresponding HS value.

181 Serum human chondroitin sulfate concentration was measured using commercial ELISA Kit 182 (Abbexa, UK). The measurement range was $3.13-200 \mathrm{ng} / \mathrm{ml}$ with $1.88 \mathrm{ng} / \mathrm{ml}$ detection limit. 183 In this case, $<8 \%$ and $<10 \%$ intra-assay and inter-assay precision can be achieved, respectively. 184 This kit is based on sandwich enzyme-linked immuno-sorbent assay technology. When using this 185 technique, an antibody specific to CS is pre-coated onto a 96-well plate. The standards, test samples and biotin detection antibody are added to the wells and rinsed with wash buffer. Biotin conjugated CS antibody serves as a detection antibody, whereas TMB substrate is used to visualize HRP enzymatic reaction. TMB is catalyzed by HRP to produce a blue-colored product that changes into yellow after adding stop solution. The intensity of the color yellow is proportional to the CS amount bound on the plate. The OD absorbance is measured spectrophotometrically at $450 \mathrm{~nm}$ in a microplate reader, allowing the CS concentration to be calculated.

Blood (serum) glucose was determined for all subjects, all of whom were given the hemoglobin A1c (HbA1c) test, to determine the average blood sugar level over the preceding three months. Serum glucose was measured using the commercial assay on Roche Cobas 6000 automated analyzer (Roche Diagnostics, Mannheim, Germany). The measurement range was 3.9-6.1 $\mathrm{mmol} / \mathrm{l}$. HbA1c analysis was performed using capillary electrophoresis technique in free solution on CapillaryFlex Piercing II instrument (Sebia, France).

199

200

201

202

203

204

205

206

207

208

209

210

211

212

213

214

215

216

217

218

219

220

221

222

\section{Statistical analysis}

Standard descriptive statistics were used (arithmetic mean with standard deviation for normally distributed numerical data, or median with range otherwise, while providing absolute and relative numbers for categorical data). Whether the data was distributed normally was established via mathematical (coefficient of variation, skewness and kurtosis, Kolmogorov-Smirnov and Shapiro-Wilk test) and graphical (histogram, normal Q-Q diagram, detrended Q-Q diagram and box-plot) methods. As at least two of the mathematical as well as graphical tests met the normal distribution criteria, we deemed this sufficient evidence for establishing that the data was normally distributed. The differences in numerical variables among the four groups were assessed via One-Way ANOVA combined with Tukey post-hoc testing, or via the KruskalWallis and Mann-Whitney U test. For testing the difference in frequencies between study groups, Chi-squared or Fisher's exact test was performed. In order to identify factors independently associated with XFG, multivariate logistic regression was performed. Models were constructed for the following inter-group comparisons: XFG vs. POAG, XFG vs. NC, and XFG vs. XFS. Model variables were selected via the "Enter" method, and VIF collinearity was examined (all variables with VIF $>5$ were eliminated from the primary model). Correlation between ophthalmic clinical parameters in XFG, POAG and XFS eyes at presentation and HS and CS concentrations in serum, as well as correlation between HbAlc and blood glucose and HS and CS concentrations was tested by examining the Spearman's correlation coefficient. Diagnosis screening tests (sensitivity, specificity) and ROC curve analysis have been applied in the determination of cut-off values for analyzed parameters.

All statistical methods were significant at $\mathrm{p} \leq 0.05$. Statistical analysis was performed in IBM SPSS ver. 21.0. 


\section{Results}

226 Demographic characteristics

227

228

229

230

231

232

233

234

235

236

237

238

239

240

241

242

243

244

245

246

247

248

249

250

251

252

253

254

255

256

257

258

259

260

261

262

263

264

265

266

267

268

269

270

The study sample comprised of 165 subjects, in 47 and 43 of whom XFG and POAG was newly diagnosed, respectively, while 22 patients had XFS and 53 individuals served as normal controls (NC). The patients' demographic characteristics and the prevalence of systemic diseases in the sample are shown in Table 1. The mean age in the XFG, POAG, XFS and NC group was $73.28 \pm$ $9.00,66.33 \pm 7.77,75.45 \pm 7.03$ and $73.45 \pm 9.49$ years, respectively, whereby the age difference was statistically significant for XFG vs. POAG $(\mathrm{p}=0.001)$ only. The age of subjects with XFM (XFG and XFS groups) was $73.97 \pm 8.43$ and was statistically significantly $(\mathrm{p}<0.001)$ higher than that $(70.26 \pm 9.41$ years) of non-XFM subjects (POAG and $\mathrm{NC}$ groups combined). Participants assigned to the XFG, POAG and NC groups were predominantly male (at $63.8 \%$, $53.5 \%$, and $62.3 \%$, respectively) while XFS subjects were predominantly female $(54.5 \%, \mathrm{p}=$ $0.150)$.

\section{Systemic diseases}

With the exception of history of coronary artery bypass or vascular surgery, prevalence of systemic diseases in the studied groups was not statistically significantly different. As shown in Table 1, while a higher percentage of patients in the XFG group reported a history of coronary artery bypass or vascular surgery compared with other groups, this difference was statistically significant for XFG vs. NC only $(21.3 \%$ vs. $7.5 \%, p=0.048)$.

\section{Ophthalmic characteristics}

The examination was performed on 330 eyes, whereby $76(23.0 \%)$ eyes pertained to newly diagnosed XFG, 86 (26.1\%) to newly diagnosed POAG, and XFS was noted in 44 (13.3\%) eyes, while in the remaining $124(37.6 \%)$ eyes, XFS or any type of glaucoma was absent, which thus served as normal control group.

The IOP in the eyes affected by XFG was higher than the IOP in the POAG eyes, whereby the difference in the mean IOP was statistically significant $(32.4 \pm 10.1 \mathrm{~mm} \mathrm{Hg}$ vs. $28.1 \pm 4.7 \mathrm{~mm}$ $\mathrm{Hg}, \mathrm{p}<0.001)$. The IOP in the XFS group $(16.3 \pm 2.6 \mathrm{~mm} \mathrm{Hg}$, $)$ was higher than in the NC group $(15.7 \pm 2.1 \mathrm{~mm} \mathrm{Hg}$, ) but the difference was not statistically significant $(\mathrm{p}=0.093)$.

Glaucoma severity indices pertaining to the newly diagnosed XFG and POAG eyes are shown in Table 2. A greater visual field loss expressed through Hodapp classification $(p=0.046)$ and MD $(p=0.024)$ was noted in eyes affected by exfoliative glaucoma relative to the POAG eyes. A statistically significantly smaller $\mathrm{vC} / \mathrm{D}$ was recorded in the POAG group $(, \mathrm{p}=0.042)$.

\section{Heparan Sulfate and Chondroitin Sulfate concentrations}

Serum human heparan sulfate concentrations (mean and standard deviation) in the XFG, POAG, $\mathrm{NC}$ and XFS groups were 3,189.0 $\pm 1,473.8 \mathrm{ng} / \mathrm{mL}, 2,091.5 \pm 940.9 \mathrm{ng} / \mathrm{mL}, 2,543.1 \pm 1,397.3$ $\mathrm{ng} / \mathrm{mL}$ and $2,658.2 \pm 1,426.8 \mathrm{ng} / \mathrm{mL}$, respectively, whereby only the differences between XFG vs. POAG $(p=0.001)$ and XFG vs. NC $(p=0.032)$ were statistically significant, while that between XFG and XFS groups was not $(\mathrm{p}=0.244)$, as shown in Fig. 1 .

Serum chondroitin sulfate concentrations (mean and standard deviation) in the XFG, POAG, NC and XFS group were $43.9 \pm 20.7 \mathrm{ng} / \mathrm{mL}, 38.5 \pm 22.0 \mathrm{ng} / \mathrm{mL}, 35.8 \pm 16.4 \mathrm{ng} / \mathrm{mL}$ and $43.3 \pm$ $21.8 \mathrm{ng} / \mathrm{mL}$ respectively. However, as shown in Fig. 2, only the difference between XFG and $\mathrm{NC}$ was statistically significant $(\mathrm{p}=0.041)$, while the difference between XFG and POAG $(\mathrm{p}=$ $0.099)$ as well as the XFG and the XFS group was not statistically significant $(p=0.857)$.

Peer) reviewing PDF | (2018:11:32496:2:0:NEW 31 Mar 2019) 
271 When the participants in the XFM (XFG and XFS) group were compared to subjects without 272 XFM (those assigned to the POAG and NC groups), their serum HS concentrations were 3,019.8 $273 \pm 1,469.8 \mathrm{ng} / \mathrm{mL}$ vs. $2,338.7 \pm 1,227.2 \mathrm{ng} / \mathrm{mL}(\mathrm{p}=0.006)$ (Fig. 3) and CS concentrations were $274 \quad 43.7 \pm 20.9 \mathrm{ng} / \mathrm{mL}$ vs. $37.1 \pm 19.0 \mathrm{ng} / \mathrm{mL}(\mathrm{p}=0.026)$ (Fig. 4$)$.

275 In Table 3 we report HS and CS concentrations segregated by gender for the XFG, POAG, XFS 276 and NC groups.

277 Factors associated with XFG that, according to multivariate logistic regression analysis, 278 distinguish it from POAG, NC, and XFS are shown in Table 4.

279 Moreover, as shown in Table 5, the Spearman's correlation coefficient values indicated absence 280 of correlation between either ophthalmic clinical parameters or indices of glaucoma severity in 281 XFG, POAG and XFS eyes at presentation and serum HS and CS concentrations.

282 Correlation between HbAlc and serum glucose and HS and CS concentrations, as measured by 283 the Spearman's correlation coefficient, is reported in Table 6.In the present study, we evaluated 284 the clinical diagnostic value of HS and CS, in terms of their sensitivity and specificity, in patients 285 with XFG compared to POAG patients, normal controls and subjects with XFS. When the XFG group was compared to NC, HS had a sensitivity of $60 \%$ and specificity of $73 \%$ at a cut-off value of 2,688.3 ng/mL. Area under the ROC curve (AUC) was $0.62(95 \% \mathrm{CI} 0.510-0.741, \mathrm{p}=$ 0.032) (Fig.5). In the comparison between XFG and POAG, HS had a sensitivity of $60 \%$ and specificity of $86 \%$ at a cut-off value of $2,642.9 \mathrm{ng} / \mathrm{mL}$, and the AUC was $0.70(95 \% \mathrm{CI}$ $0.590-0.814, \mathrm{p}=0.001)$. In the comparison of XFG to all remaining groups, HS had a sensitivity of $64 \%$ and specificity of $71 \%$ at a cut-off value of $2,495.4 \mathrm{ng} / \mathrm{mL}$, with the AUC of $0.65(\mathrm{p}=$ 0.003 ), and if the group with XFM was compared to the one without XFM, HS had a sensitivity of $59 \%$ and specificity of $74 \%$ at a cut-off value of $2,495.4 \mathrm{ng} / \mathrm{mL}$, with the AUC of $0.63(95 \%$ CI $0.538-0.721, \mathrm{p}=0.006$ ).

With respect to the diagnostic value of CS, if XFG was compared to NC, its sensitivity and specificity was $60 \%$ and $62 \%$, respectively, at a cut-off value of $3.92 \mathrm{ng} / \mathrm{mL}$, while the AUC was $0.62(95 \%$ CI $0.508-0.730, p=0.041)$ (Fig.6). When the group with XFM was compared to the one without XFM, CS had a sensitivity of $64 \%$ and specificity of $61 \%$ at a cut-off value of 3.62 $\mathrm{ng} / \mathrm{mL}$, with the AUC of 0.60 (95\% CI 0.513-0.690, $\mathrm{p}=0.026)$.

If serum HS and CS concentrations were measured simultaneously, the sensitivity and specificity values were $84 \%$ and $45 \%$, respectively, when the XFG group was compared to $\mathrm{NC}$, whereas if the group with XFM was compared to that without XFM, a sensitivity of $85 \%$ and a specificity of $44 \%$ were obtained.

\section{Discussion}

Exfoliation syndrome is age-related disorder characterized by the pathological accumulation deposition of fibrillar material in multiple tissues, frequently associated with severe secondary open-angle glaucoma (Puska, 2015; Ritch \& Schlötzer-Schrehardt, 2001).

XFG typically develops after 60 years of age and, in most cases, significant optic nerve head and visual field damage in at least one eye is already present at the time of diagnosis (Ritch \& Schlötzer-Schrehardt, 2001). XFG clinical features are distinct from those characterizing POAG (Gonzalez-Iglesias et al., 2014).

In the present study, XFG patients were statistically significantly older than those diagnosed with POAG, as their mean age was 7 years greater, but they were of similar age as the XFS participants. 
317 The visual acuity findings yielded by our study indicate a more severe visual loss in newly 318 diagnosed XFG eyes relative to that noted in the POAG group. Compared with the POAG group, 319 higher mean IOP was measured in the XFG eyes that took part in the present study.

320 Moreover, a significantly greater severity of optic nerve damage was noted in newly diagnosed 321 XFG compared to newly diagnosed POAG, as indicated by $\mathrm{vC} / \mathrm{D}$. In addition, the newly 322 diagnosed XFG patients also had significantly more advanced visual field changes than those 323 newly diagnosed with POAG.

324 As early diagnosis is key to preventing visual impairment in glaucoma, especially in XFG which 325 has aggressive course, extensive research efforts have recently been dedicated to glaucoma 326 In principle, a biomarker is an indicator of a biochemical feature or facet that can be used to diagnose or monitor the progress of a disease (Ross et al., 2005). Thus, an agent of measureable entity with high sensitivity and specificity that accurately predicts the presence, progression, or absence of a disease would be an "ideal" biomarker. Hence, in XFS subjects, it is critical that a biomarker can accurately and reliably predict progression from syndrome to glaucoma, as well as allow identification of individuals at high risk of progressive damage. Given that XFS is a systemic disease, serum or plasma would be ideal bodily fluids for identification of potential biomarkers. Thus far, systemic exfoliation material has never been diagnosed in the absence of intraocular manifestations (Ritch \& Schlötzer-Schrehardt, 2001).

Human serum or plasma serve as typical clinical specimens, since both are accessible and convenient for use in clinical trials. It has been demonstrated that the low molecular weight fraction of human serum or plasma provides a rich source of potential biomarkers pertaining to diseases generated through enzymatic cleavage (Anderson et al., 2004; Adkins et al., 2002). The aim of the present study was to investigate with relatively simple method, patients' serum samples, in order to examine potential biomarkers (GAG concentrations, chondroitin sulfate and heparan sulfate) in newly diagnosed untreated XFG and compare their levels with those pertaining to newly diagnosed untreated POAG, as well as normal controls and subjects with XFS. To the best of our knowledge, no prior studies on this topic have been conducted to date. CS and HS are two distinct classes of glycosaminoglycans. Both are distributed on the surface of virtually all cells and throughout most extracellular matrices. Consequently, CS and HS are the major GAGs in the blood, whereby other serum GAGs include keratin sulfate and hyaluronan (Lu et al., 2010). GAGs such as CS and HS in human serum or plasma carry important biological information and their variations in human serum or plasma are vital for investigating and monitoring certain disease conditions, such as cancer, rheumatoid arthritis and diabetes mellitus (DM) (Li et al., 2017; Pothacharoen et al., 2006; Lamari et al., 2006; Komosińska-Vassev et al., 2005). Therefore, evaluating GAG presence and quantity variations has a great potential for disease diagnosis and prognosis (Wei et al., 2011). Nevertheless, no standardized methods exist for serum/plasma GAG isolation and quantification ( $\mathrm{Lu}$ et al., 2010). The approach based on a sandwich ELISA adopted in the present study was easy to perform, and it yielded reliable and reproducible quantitative results.

In the current study, HS serum concentration in newly diagnosed XFG patients was significantly higher relative to that in newly diagnosed POAG patients and NC. While elevated concentrations were also noted for the XFG group relative to the subjects with XFS, the difference was not statistically significant. Similarly, serum CS concentrations in newly diagnosed XFG patients were elevated compared to the normal controls, as well as POAG and XFS subjects; however, only the XFG vs. NC difference was statistically significant. 
363 As already mentioned, XFS is a generalized disorder of the ECM characterized by production 364 and progressive accumulation of XFM deposits in tissues of the anterior segment, as well a 365 systemic disorder. These findings suggest that ocular XFS is part of a general disorder of the 366 extracellular matrix and that patients with XFM may suffer from increased comorbidity. As $367 \mathrm{XFS} / \mathrm{XFG}$ results in excessive pathological ECM deposition, this increases GAG, as well as 368 heparan and chondroitin sulfate levels.

369 When the CS values were examined with respect to gender, they were higher in women relative 370 to men in the XFG, POAG and NC groups, but the difference was statistically significant in the 371 POAG group only. XFS was the only group in which male subjects had negligibly higher CS 372 values. On the other hand, HC concentration values were higher among men in XFG, NC and 373 XFS groups, but the difference relative to women was not statistically significant. Slightly higher 374 HC values were obtained for women in the POAG group.

375 The multivariate analysis findings revealed that, GAG concentrations (especially HS) were 376 associated with XFG. In addition, statistically significant differences in the serum concentrations 377 of GAGs, HS and CS were noted between XFM patients (XFG and XFS group) and subjects 378 without XFM (those assigned to the POAG and NC groups).

379 The total amount of GAGs in serum was previously found to be closely related to diabetic 380 pathology, advanced diabetic complications in particular (Komosińska-Vassev et al., 2005). 381 However, in our study, presence of DM did not influence the results, and the differences among 382 groups were not statistically significant. Moreover, in line with the results reported by Shingleton et al. (2003), DM was slightly less frequent in the exfoliation group relative to the nonexfoliation group. Similarly, Musch et al. (2012) found diabetes significantly more frequent among patients with POAG (18.2\%) vs. patients with XFG (3.5\%). In an earlier study, Konstas et al. (1998) noted a lower prevalence of diabetes in patients with XFG requiring surgery than in those with POAG. Thus, in multivariate logistic regression analyses, we adjusted for presence of diabetes mellitus. On the other hand, blood glucose and HbAlc values were not statistically significantly different across groups. No link between blood glucose and HbA1c and HS and CS values was noted in the XFG group, while in the $\mathrm{NC}$ group heparan sulfate and blood glucose were in the moderately positive statistically significant correlation, whereas in the XFS group HbAlc was in a negative moderate correlation with chondroitin sulfate.

Identification of potential XFG biomarkers from bodily fluids has been the subject of extensive research. For example, McNally \& O'Brien (2014) highlighted some important findings yielded by employing metabolomics and proteomics strategies in the search for possible biomarkers of XFG from anterior lens capsule, blood or aqueous humor in exfoliation glaucoma patients. Similarly, Kamel \& Bourke \& O’Brien (2018) conducted a literature review in order to present an up-to-date list of clinical and laboratory-based biomarkers relating to exfoliation syndrome and exfoliative glaucoma.

In prior studies in this field, analyses were time consuming and complex in most cases, involving analysis possible biomarkers from the anterior lens capsules, TM and the aqueous humor. Ghanem \& Arafa \& El-Baz (2011) analyzed the aqueous humor composition in XFS/XFG patients because all ocular tissues involved are bathed by the aqueous humor and should therefore be influenced by the factors contained therein. However, the practical utility of these findings is limited, as aqueous humor cannot be easily obtained in routine clinical practice.

Gonzalez-Iglesias et al. (2014) conducted a comparative proteomic study involving serum of patients with POAG and XFG to identify a candidate panel of glaucoma biomarkers for the clinical prediction, prognosis, diagnosis and monitoring of POAG and XFG cases. 
409 The novel contribution of the present study stems from attempting to establish a link between HS 410 and CS serum concentrations with ophthalmic clinical parameters in XFG, XFS and POAG eyes, 411 which was not supported by the research findings. Moreover, no correlation was noted between 412 HS and CS serum concentrations and glaucomatous damage in newly diagnosed XFG and 413 POAG cases, expressed via glaucoma severity indices, such as vC/D, Hodapp Classification and 414 MD.

415 Our aim was to identify a biomarker that would be specific to patients with XFG. Our results as 416 well as findings reported by other authors indicate that, in XFG, significant optic nerve head and 417 visual field damage is already present at the time of diagnosis (Ritch \& Schlötzer-Schrehardt, 418 2001; Konstas et al., 2006). Consequently, in the involved eye(s), optic nerve head damage and 419 visual filed deterioration are frequently more severe in XFG compared to POAG patients (Ritch, 420 2001). Owing to these facts, we attempted to discover serum biomarkers that would prompt a 421 patient to seek ophthalmologist consultation, if indicated by the blood analysis results, in order to 422 obtain a timely XFG diagnosis and start appropriate therapy. This is highly relevant, given that 423 part of the present investigation, we evaluated the clinical diagnostic value of HS and CS and, according to our findings, we established that HS is an adequate diagnostic test for comparing $\mathrm{XFG}$ to NC and all other control groups, whereas CS is an adequate diagnostic test for comparing XFG with NC. Moreover, these tests, when performed separately, can adequately distinguish subjects with XFM from those without XFM. Clearly, performing both tests would be advantageous, as this would increase sensitivity. It should be noted that the results reported here were affected by a small sample size, as noted in study limitations.

When interpreting the results yielded by our investigation, it is important to note some study limitations. Specifically, as the study aim was to determine the time of the XFG and POAG occurrence, we did not select POAG patients that were age- and sex-matched to the XFG cohort. The existence of systemic diseases was established through interviews, as well as via a detailed review of medical documentation. Thus, it needs to be emphasized that systemic diseases were not clinically determined in our study sample. It is also noteworthy that we might have failed to detect some subclinical cases of XFS, which would be diagnosable by histological methods only. Moreover, DM patients were not excluded from the sample, as already explained, and we adjusted for presence of diabetes mellitus in multivariate logistic regression analyses. Our findings confirmed absence of statistically significant differences between groups, indicating that inclusion of DM patients should not have influenced our results. A further limitation of the study is a small sample size.

\section{Conclusions}

In conclusion, our findings revealed greater HS and CS concentrations in newly diagnosed XFG patients and XFS subjects compared to those without XFM. HS is an adequate diagnostic test for comparing XFG to $\mathrm{NC}$ and all other control groups, whereas CS is an adequate diagnostic test for comparing XFG with NC.

As this work, to the best of our knowledge, marks the first attempt to evaluate HS and CS serum concentrations in both XFG and XFS subjects, this data and ongoing research in this field will continue to improve our understanding of XFS/XFG as a systemic disorder. Implications of HS and CS in the pathophysiology of exfoliation syndrome and glaucoma should be studied further. Serum or plasma are easily accessible and should thus be explored as rich sources of potential 
454 disease biomarkers. Further research should aim to identify XFG biomarkers that could be

455

456

457

458

459

460

461

462

463

464

465

466

467

468

469

470

471

472

473

474

475

476

477

478

479

480

481

482

483

484

485

486

487

488

489

490

491

492

493

494

495

496

497

498

499

utilized in routine blood analysis tests, aiding in timely disease diagnosis.

\section{References}

1. Weinreb RN, Aung T, Medeiros FA. 2014. The pathophysiology and treatment of glaucoma: a review. JAMA 14;311(18):1901-11 DOI: 10.1001/jama.2014.3192

2. Quigley HA, Broman AT. 2006. The number of people with glaucoma worldwide in 2010 and 2020. British Journal of Ophthalmology 90:262-7 DOI:10.1136/bjo.2005.081224

3. Prum BE Jr, Rosenberg LF, Gedde SJ, Mansberger SL, Stein JD, Moroi SE, Herndon LW Jr, Lim MC, Williams RD. 2016. Primary Open-Angle Glaucoma Preferred Practice Pattern(®) Guidelines. Ophthalmology 123(1):P41-P111

DOI: 10.1016/j.ophtha.2015.10.053.

4. Hollo G, Katsanos A, Konstas AG. 2015. Management of exfoliative glaucoma: challenges and solutions. Clinical Ophthalmology 9:907-19

DOI: $10.2147 /$ OPTH.S77570

5. Ritch R, Schlötzer-Schrehardt U, Konstas AG. 2003. Why is glaucoma associated with exfoliation syndrome? Progress in Retinal and Eye Research 22(3):253-75

6. Ritch R, Schlötzer-Schrehardt U. 2001. Major review: Exfoliation syndrome. Survey of Ophthalmology 45(4):265-315

7. Ritch R. 2015. From exfoliation syndrome to exfoliative glaucoma. In: Hollo G, Konstas AGP, editors. Exfoliation Syndrome and Exfoliative Glaucoma. 3nd ed. Savona, Italy: Publicomm Srl, 19-23

8. Puska P. 2015. Development of exfoliative glaucoma. In: Hollo G, Konstas AGP, editors. Exfoliation Syndrome and Exfoliative Glaucoma. 3nd ed. Savona, Italy: Publicomm Srl, 133-136

9. Zenkel M, Schlötzer-Schrehardt U. 2014. The composition of exfoliation material and the cells involved in its production. Journal of Glaucoma 23(8 Suppl 1):S12-4 DOI:10.1097/IJG.0000000000000123

10. Schlötzer-Schrehardt U, Naumann GO. 2015. Morphology of exfoliation syndrome. In: Holló G, Konstas AGP, editors. Exfoliation Syndrome and Exfoliative Glaucoma. 3nd ed. Savona, Italy: Publicomm Srl, 35-42

11. Yue B. 2014. Biology of the extracellular matrix: an overview. Journal of Glaucoma 23(8 Suppl 1):S20-23 DOI:10.1097/IJG.0000000000000108

12. Huflejt ME, Preiss JS, Thomas JE, Gils IM, Vuskovic MI. 2014. Glycomics, extracellular matrix, and antiglycan antibodies in exfoliation syndrome. Journal of Glaucoma 23(8 Suppl 1):S24-29 DOI: 10.1097/IJG.0000000000000118

13. Schlötzer-Schrehardt U, Naumann GO. 2006. Ocular and systemic pseudoexfoliation syndrome. American Journal of Ophthalmology 141:921-937 DOI:10.1016/j.ajo.2006.01.047

14. Schlötzer-Schrehardt U. 2012. Pseudoexfoliation syndrome: the puzzle continues. Journal of Ophthalmic and Vision Research 7:187-189

15. Baba H. 1983. Histochemical and polarization optical investigation for glycosaminoglycans in exfoliation syndrome. Graefe's Archive for Clinical and Experimental Ophthalmology 221:106-109 
500

501

502

503

504

505

506

507

508

509

510

511

512

513

514

515

516

517

518

519

520

521

522

523

524

525

526

527

528

529

530

531

532

533

534

535

536

537

538

539

540

541

542

543

544

16. Schlötzer-Schrehardt U, Dorfler S, Naumann GO. 1992. Immunohistochemical localization of basement membrane components in pseudoexfoliation material of the lens capsule. Current Eye Research 11:343-355

17. Lu H, McDowell LM, Studelska DR, Zhang L. 2010. Glycosaminoglycans in Human and Bovine Serum: Detection of Twenty-Four Heparan Sulfate and Chondroitin Sulfate Motifs Including a Novel Sialic Acid modified Chondroitin Sulfate Linkage Hexasaccharide. Glycobiology Insights 2:13-28

18. Li X, Lan Y, He Y, Liu Y, Luo H, Yu H, Song N, Ren S, Liu T, Hao C, Guo Y, Zhang L. 2017. Heparan Sulfate and Chondroitin Sulfate Glycosaminoglycans Are Targeted by Bleomycin in Cancer Cells. Cellular Physiology and Biochemistry 43(3):1220-1234 DOI:10.1159/000481763

19. Pothacharoen P, Teekachunhatean S, Louthrenoo W, Yingsung W, Ong-Chai S, HardinghamT, Kongtawelert P. 2006. Raised chondroitin sulfate epitopes and hyaluronan in serum from rheumatoid arthritis and osteoarthritis patients. Osteoarthritis and Cartilage 14(3):299-301

DOI:10.1016/j.joca.2005.10.005

20. Gonzalez-Iglesias H, Alvarez L, Garcia M, Escribano J, Rodriguez-Calvo PP, FernandezVega L, CocaPrados M. 2014. Comparative proteomic study in serum of patients with primary open-angle glaucoma and pseudoexfoliation glaucoma. Journal of Proteomics 98:65-78 DOI:10.1016/j.jprot.2013.12.006

21. Ross JS, Symmans WF, Pusztai L, Hortobagyi GN. 2005. Pharmacogenomics and clinical biomarkers in drug discovery and development. American Journal of Clinical Pathology 124(Suppl.1):S29-S41 DOI:10.1309/XYQAFANAPYNC6X59

22. Anderson NL, Polanski M, Pieper R, Gatlin T, Tirumalai RS, Conrads TP, Veenstra TD, Adkins JN, Pounds JG, Fagan R, Lobley A. 2004. The human plasma proteome: a nonredundant list developed by combination of four separate sources. Molecular \& Cellular Proteomics 3(4):311-326 DOI:10.1074/mcp.M300127-MCP200

23. Adkins JN, Varnum SM, Auberry KJ, Moore RJ, Angell NH, Smith RD, Springer DL, Pounds JG. 2002. Toward a human blood serum proteome: analysis by multidimensional separation coupled with mass spectrometry. Molecular \& Cellular Proteomics 1(12):947-955 DOI:10.1074/mcp. M200066-MCP200

24. Lamari FN, Theocharis AD, Asimakopoulou AP, Malavaki CJ, Karamanos NK. 2006. Biomedical Chromatography 20:539-550 DOI:10.1002/bmc.669

25. Komosińska-Vassev K, Olczyk K, Koźma EM, Olczyk P, Wisowski G, Winsz-Szczotka K. 2005. Alterations of glycosaminoglycan metabolism in the development of diabetic complications in relation to metabolic control. Clinical Chemistry and Laboratory Medicine 43(9):924-9 DOI:10.1515/CCLM.2005.158

26. Wei W, Niñonuevo MR, Sharma A, Danan-Leon LM, Leary JA. 2011. A comprehensive compositional analysis of heparin/heparin sulfate derived disaccharides from human serum. Analyltical Chemistry 83(10):3703-8 DOI:10.1021/ac2001077

27. Shingleton BJ, Heltzer J, O'Donoghue MW. 2003. Outcomes of phacoemulsification in patients with and without pseudoexfoliation syndrome. Journal of Cataract and Refractive Surgery 29(6):1080-6

28. Musch DC, Shimizu T, Niziol LM, Gillespie BW, Cashwell LF, Lichter PR. 2012. Clinical characteristics of newly diagnosed primary, pigmentary andpseudoexfoliat 
545

546

547

548

549

550

551

552

553

554

555

556

557

558

559

560

561

562

563

564

ive open-angle glaucoma in the Collaborative Initial Glaucoma Treatment Study. British Journal of Ophthalmology 96(9):1180-4 DOI: 10.1136/bjophthalmol-2012-301820

29. Konstas AGP, Tsatsos I, Kardasopoulos A, Bufidis T, Maskaleris G. 1998. Preoperative features of patients with exfoliation glaucoma and primary open-angle glaucoma. The AHEPA study. Acta Ophthalmologica(Copenh) 76:208-12

30. McNally S, O'Brien CJ. 2014. Metabolomics/Proteomics strategies used to identify biomarkers for exfoliation glaucoma. Journal of Glaucoma 23(8 Suppl 1):S51-54 DOI: 10.1097/IJG.0000000000000117

31. Kamel K, Bourke L, O'Brien C. 2018. Clinical and Laboratory Biomarkers for Pseudoexfoliation Syndrome. Journal of Glaucoma 27(Suppl 1):S111-S113DOI: 10.1097/IJG.0000000000000924

32. Ghanem AA, Arafa LF, El-Baz A. 2011. Connective tissue growth factor and tissue inhibitor of matrixmetalloproteinase-2 in patients with exfoliative glaucoma. Current Eye Research 36(6):540-5 DOI:10.3109/02713683.2011.565541

33. Konstas AGP, Hollo G, Astakhov YS, Teus MA, Akopov EL, Jenkins JN, Stewart WC. 2006. Presentation and long-term follow-up of exfoliation glaucoma in Greece, Spain, Russia, and Hungary. European Journal of Ophthalmology 16(1):60-66

34. Ritch R. 2001. Perspective on exfoliation syndrome. Journal of Glaucoma 10(5 Supp1 1):S33-S35. 


\section{Table $\mathbf{1}$ (on next page)}

Demographic characteristics and systemic diseases of subjects with XFG, POAG, XFS and NC

XFG: exfoliative glaucoma; POAG: primary open-angle glaucoma; NC: normal controls; XFS:

controls with exfoliation syndrome; $n *$ : number of patients; $y$ : years; DM: diabetes mellitus; SH: systemic hypertension; MS: myocardial stroke; CAB: coronary artery bypass; VS: vascular surgery; AAA: abdominal aortic aneurysm; ACD: acute cerebrovascular disease; *Statistically

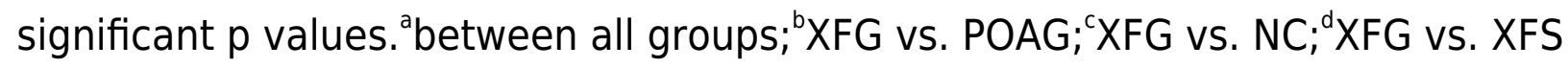




\section{Table 1:}

2 Demographic characteristics and systemic diseases of subjects with XFG, POAG, XFS and NC

\begin{tabular}{lcccccccc}
\hline Characteristic & $\begin{array}{c}\text { XFG } \\
\left(\mathrm{n}^{*}=47\right)\end{array}$ & $\begin{array}{c}\text { POAG } \\
\left(\mathrm{n}^{*}=43\right)\end{array}$ & $\mathbf{p}^{\mathbf{b}}$ & $\begin{array}{c}\mathbf{N C} \\
\left(\mathrm{n}^{*}=53\right)\end{array}$ & $\mathbf{p}^{\mathbf{c}}$ & $\begin{array}{c}\text { XFS } \\
\left(\mathrm{n}^{*}=22\right)\end{array}$ & $\mathbf{p}^{\mathbf{d}}$ & $\mathbf{p}^{\mathbf{a}}$ \\
\hline Age, mean \pm SD(y) & $73.28 \pm 9.00$ & $66.33 \pm 7.77$ & $0.001^{*}$ & $73.45 \pm 9.49$ & 1.000 & $75.45 \pm 7.03$ & 0.746 & $<0.001^{*}$ \\
Male gender, n (\%) & $30(63.8)$ & $23(53.5)$ & 0.319 & $33(62.3)$ & 0.871 & $10(45.5)$ & 0.150 & 0.418 \\
DM, n (\%) & $12(25.5)$ & $15(34.9)$ & 0.334 & $15(28.3)$ & 0.755 & $6(27.3)$ & 0.878 & 0.792 \\
HbA1c, (\%) & $5.72 \pm 0.50$ & $6.02 \pm 0.89$ & 0.424 & $5.91 \pm 0.79$ & 0.656 & $5.83 \pm 0.83$ & 0.908 & 0.439 \\
Blood glucose & $5.69 \pm 1.67$ & $6.28 \pm 2.82$ & 0.617 & $6.25 \pm 2.29$ & 0.504 & $5.61 \pm 1.38$ & 0.997 & 0.251 \\
SH, n (\%) & $36(76.6)$ & $27(62.8)$ & 0.153 & $38(71.7)$ & 0.577 & $16(72.7)$ & 0.473 & 0.539 \\
MS, n (\%) & $4(8.5)$ & $1(2.3)$ & 0.201 & $2(3.8)$ & 0.319 & $1(4.5)$ & 0.554 & 0.553 \\
Arrhythmia, n (\%) & $12(25.5)$ & $5(11.6)$ & 0.092 & $12(22.6)$ & 0.736 & $4(18.2)$ & 0.500 & 0.383 \\
CAB or VS, n (\%) & $10(21.3)$ & $4(9.3)$ & 0.117 & $4(7.5)$ & $0.048 *$ & $3(13.6)$ & 0.449 & 0.184 \\
AAA, n (\%) & $3(6.4)$ & $2(4.7)$ & 0.720 & $0(0)$ & 0.062 & $0(0)$ & 0.546 & 0.226 \\
ACD, n (\%) & $3(6.4)$ & $2(4.7)$ & 0.720 & $3(5.7)$ & 0.879 & $1(4.5)$ & 0.761 & 0.982 \\
\hline XFG: $2 x(\%)$
\end{tabular}

XFG: exfoliative glaucoma; POAG: primary open-angle glaucoma; NC: normal controls; XFS: controls with exfoliation syndrome; $n^{*}$ : number of patients; y: years; DM: diabetes mellitus; SH: systemic hypertension; MS: myocardial stroke; CAB: coronary artery bypass; VS: vascular surgery; AAA: abdominal aortic aneurysm; ACD: acute cerebrovascular disease; * Statistically significant $\mathrm{p}$ values.

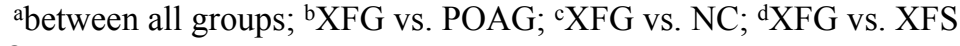

3

4

5

6

7

8

9

10

11

12

13

14

15

16

17

18

19

20

21

22

23

24

25

26

27

28 


\section{Table 2 (on next page)}

Clinical features and indices of glaucoma severity in XFG and POAG eyes at presentation

BCVA: best-corrected visual acuity; LP: light perception; NLP: no light perception; IOP:

intraocular pressure; MD: mean deviation; PSD: pattern standard deviation; VC/D: vertical cup to disc ratio; $n *$ : number of eyes; *Statistically significant $p$ values. 
1 Table 2:

2 Clinical features and indices of glaucoma severity in XFG and POAG eyes at presentation

\begin{tabular}{|c|c|c|c|}
\hline Characteristic & $\begin{array}{c}\mathbf{X F G} \\
\left(\mathrm{n}^{*}=76\right)\end{array}$ & $\begin{array}{l}\text { POAG } \\
(n *=86)\end{array}$ & $\mathbf{p}$ \\
\hline $\begin{array}{l}\text { BCVA, } \\
\text { med (min-max) }\end{array}$ & $0.70(0.008-1.0)$ & $1.0(0.03-1.0)$ & $<0.001^{*}$ \\
\hline $\mathrm{LP}, \mathrm{n}(\%)$ & $2(1.8)$ & $1(0.7)$ & \\
\hline NLP, n $(\%)$ & $3(2.6)$ & $0(0)$ & \\
\hline $\begin{array}{l}\text { IOP }(\mathrm{mmHg}) \\
\text { mean } \pm \text { SD }\end{array}$ & $32.4 \pm 10.1$ & $28.1 \pm 4.7$ & $<0.001 *$ \\
\hline Hodapp, n (\%) & & & $0.046^{*}$ \\
\hline early & $31(40.8)$ & $53(61.7)$ & \\
\hline moderate & $16(21.1)$ & $15(17.4)$ & \\
\hline advanced & $19(25.0)$ & $13(15.1)$ & \\
\hline without visual field & $10(13.1)$ & $5(5.8)$ & \\
\hline MD, med (min-max) & $-5.26(-1.58$ to -28.27$)$ & $-4.13(-1.39$ to -31.02$)$ & $0.024 *$ \\
\hline PSD, med (min-max) & $4.05(1.55-12.43)$ & $3.02(1.46-14.9)$ & 0.128 \\
\hline $\mathrm{vC} / \mathrm{D}$, med (min-max) & $0.6(0.45-1.0)$ & $0.5(0.45-1.0)$ & $0.042 *$ \\
\hline
\end{tabular}

3

4

5

6

7

8

9

10

11

12

13

14

15

16

17

Peer] reviewing PDF | (2018:11:32496:2:0:NEW 31 Mar 2019) 


\section{Table 3 (on next page)}

HS and CS concentrations segregated by gender for the XFG, POAG, XFS and NC subjects

HS: heparan sulfate; CS: chondroitin sulfate; M: male gender; F: female gender; *Statistically significant $p$ values 
1 Table 3:

2 HS and CS concentrations segregated by gender for the XFG, POAG, XFS and NC subjects

\begin{tabular}{|c|c|c|c|c|c|c|c|c|c|}
\hline & & XFG & $\mathrm{p}$ & POAG & $\mathrm{p}$ & $\mathrm{NC}$ & $\mathrm{p}$ & XFS & $\mathrm{p}^{*}$ \\
\hline \multirow{2}{*}{$\mathrm{CS}$} & $\mathrm{M}$ & $41.98 \pm 19.97$ & 0.413 & $29.36 \pm 14.37$ & $0.008^{*}$ & $34.75 \pm 15.68$ & 0.660 & $43.30 \pm 24.43$ & 1.000 \\
\hline & $\mathrm{F}$ & $47.23 \pm 22.13$ & & $49.09 \pm 24.76$ & & $37.67 \pm 17.72$ & & $43.21 \pm 21.46$ & \\
\hline HS & $\begin{array}{l}\mathrm{M} \\
\mathrm{F}\end{array}$ & $\begin{array}{l}3196.7 \pm 1562.0 \\
3175.5 \pm 1349.9\end{array}$ & 0.859 & $\begin{array}{l}1800.4 \pm 488.7 \\
2425.9 \pm 1208.6\end{array}$ & 0.242 & $\begin{array}{l}2579.4 \pm 1317.3 \\
2477.7 \pm 1520.4\end{array}$ & 0.419 & $\begin{array}{l}3172.9 \pm 1396.1 \\
2229.3 \pm 1360.2\end{array}$ & 0.140 \\
\hline
\end{tabular}

3 HS: heparan sulfate; CS: chondroitin sulfate; M: male gender; F: female gender;

$4 \quad$ *Statistically significant $p$ values 


\section{Table 4 (on next page)}

Factors associated with XFG vs. POAG, NC, XFS according to multivariate logistic regression analysis

BCVA: best-corrected visual acuity; IOP: intraocular pressure; MD: mean deviation; VC/D: vertical cup to disc ratio; HS: heparan sulfate; CS: chondroitin sulfate; CAB: coronary artery bypass; VS: vascular surgery; *Statistically significant $p$ values. $p^{a}$ : adjusted for age and gender; $\mathrm{p}^{\mathrm{b}}$ : adjusted for age and gender and presence of diabetes mellitus 
1 Table 4:

2 Factors associated with XFG vs. POAG, NC, XFS according to multivariate

3 logistic regression analysis

\begin{tabular}{lcccc}
\hline \multicolumn{5}{c}{ Multivariate logistic regression analysis } \\
\hline XFG vs. POAG & OR, 95\% CI OR & $\mathrm{p}$ & $\mathrm{p}^{\mathrm{a}}$ & $\mathrm{p}^{\mathrm{b}}$ \\
Age & $0.989,0.91-1.07$ & 0.787 & $/$ & $/$ \\
BCVA & $307.52,5.47-172850$ & $0.005^{*}$ & $0.002^{*}$ & $0.002^{*}$ \\
IOP & $1.979,0.85-4.60$ & 0.113 & 0.142 & 0.133 \\
Hodapp & $0.431,0.21-0.87$ & $0.018^{*}$ & $0.018^{*}$ & $0.019^{*}$ \\
MD & $0.846,0.75-0.96$ & $0.009^{*}$ & $0.002^{*}$ & $0.003^{*}$ \\
vC/D & $0.953,0.87-1.05$ & 0.320 & 0.164 & 0.198 \\
HS & $0.999,0.99-1.00$ & $0.047^{*}$ & $0.022^{*}$ & $0.028^{*}$ \\
XFG vs. NC & & & & \\
CAB or VS & $6.457,0.92-45.45$ & 0.061 & $0.046^{*}$ & 0.060 \\
MD & $0.858,0.31-2.39$ & 0.770 & 0.996 & 0.831 \\
vC/D & $0.257,0.03-2.09$ & 0.204 & 0.274 & 0.248 \\
HS & $1.000,0.99-1.00$ & 0.081 & $0.045^{*}$ & $0.046^{*}$ \\
CS & $0.855,0.59-1.24$ & 0.095 & 0.091 & 0.065 \\
XFG vs. XFS & & & & \\
vC/D & $1.016,1.00-1.03$ & 0.123 & 0.118 & 0.129 \\
MD & $1.042,0.28-3.94$ & 0.951 & 0.579 & 0.963 \\
\hline
\end{tabular}

BCVA: best-corrected visual acuity; IOP: intraocular pressure; MD: mean deviation; vC/D: vertical cup to disc ratio; HS: heparan sulfate; $\mathrm{CS}$ : chondroitin sulfate; CAB: coronary artery bypass; VS: vascular surgery; *Statistically significant $\mathrm{p}$ values.

$\mathrm{p}^{\mathrm{a}}$ : adjusted for age and gender; $\mathrm{p}^{\mathrm{b}}$ : adjusted for age and gender and presence of diabetes mellitus

4

5

6

7

8

9

10

11

12

13

14

15

16

17

18

19

20

21

22 


\section{Table 5 (on next page)}

Correlation coefficients between clinical parameters and indices of glaucoma severity at presentation and HS and CS concentrations

No correlation in all examined parameters, Spearman's correlation coefficient BCVA: bestcorrected visual acuity; IOP: intraocular pressure; MD: mean deviation; VC/D: vertical cup to disc ratio; HS: heparan sulfate; CS: chondroitin sulfate 
1 Table 5:

2 Correlation coefficients between clinical parameters and indices of glaucoma severity at presentation and HS and CS 3 concentrations

\begin{tabular}{lcccccc}
\hline & \multicolumn{2}{c}{ XFG } & \multicolumn{2}{c}{ POAG } & \multicolumn{2}{c}{ XFS } \\
\hline Parameters & HS & CS & HS & CS & HS & CS \\
\hline BCVA & -0.09 & -0.14 & -0.20 & -0.02 & -0.15 & -0.20 \\
IOP & 0.03 & 0.22 & -0.10 & -0.19 & -0.06 & -0.19 \\
Hodapp & -0.10 & 0.11 & -0.13 & -0.12 & $/$ & $/$ \\
MD & -0.17 & -0.30 & 0.13 & 0.30 & 0.24 & 0.42 \\
vC/D & 0.08 & 0.05 & 0.09 & -0.17 & 0.24 & -0.33 \\
\hline
\end{tabular}

No correlation in all examined parameters, Spearman's correlation coefficient

BCVA: best-corrected visual acuity; IOP: intraocular pressure; MD: mean deviation; vC/D: vertical cup to disc ratio; HS: heparan sulfate; CS: chondroitin sulfate

4

5

6

7

8 
Table 6(on next page)

Correlation coefficients between $\mathrm{HbAlc}$ and blood glucose and HS and CS concentrations

Spearman rank correlation coefficient HS: heparan sulfate; CS: chondroitin sulfate; *Statistically significant $p$ values 
1 Table 6:

2 Correlation coefficients between HbA1c and blood glucose and HS and

3 CS concentrations

\begin{tabular}{ccc}
\hline \multirow{2}{*}{ Group } & \multicolumn{2}{c}{ Variable coefficient value $(\mathrm{p})$} \\
HbAlc \\
\hline BFG & $-0.05(0.720)$ & $-0.24(0.105)$ \\
HS & $-0.01(0.933)$ & $0.00(0.998)$ \\
HS & & \\
POAG & $-0.17(0.273)$ & $-0.28(0.063)$ \\
CS & $-0.21(0.168)$ & $-0.07(0.680)$ \\
HS & & \\
NC & $0.02(0.900)$ & $0.03(0.819)$ \\
CS & $0.36(0.008)^{*}$ & $0.25(0.073)$ \\
HS & & $-0.48(0.024)^{*}$ \\
XFS & $-0.18(0.426)$ & $-0.31(0.157)$ \\
CS & $-0.18(0.422)$ & \\
HS &
\end{tabular}

4 Spearman rank correlation coefficient HS: heparan sulfate; CS: chondroitin

5 sulfate; $*$ Statistically significant $\mathrm{p}$ values 
Figure 1

Serum Heparan Sulfate concentrations, ng/mL in subjects with XFG, POAG, NC, XFS.

Box plots present median, bars are minimum and maximum values, circles are outliers and stars are extreme values. subjects with XFG, POAG, NC, XFS.

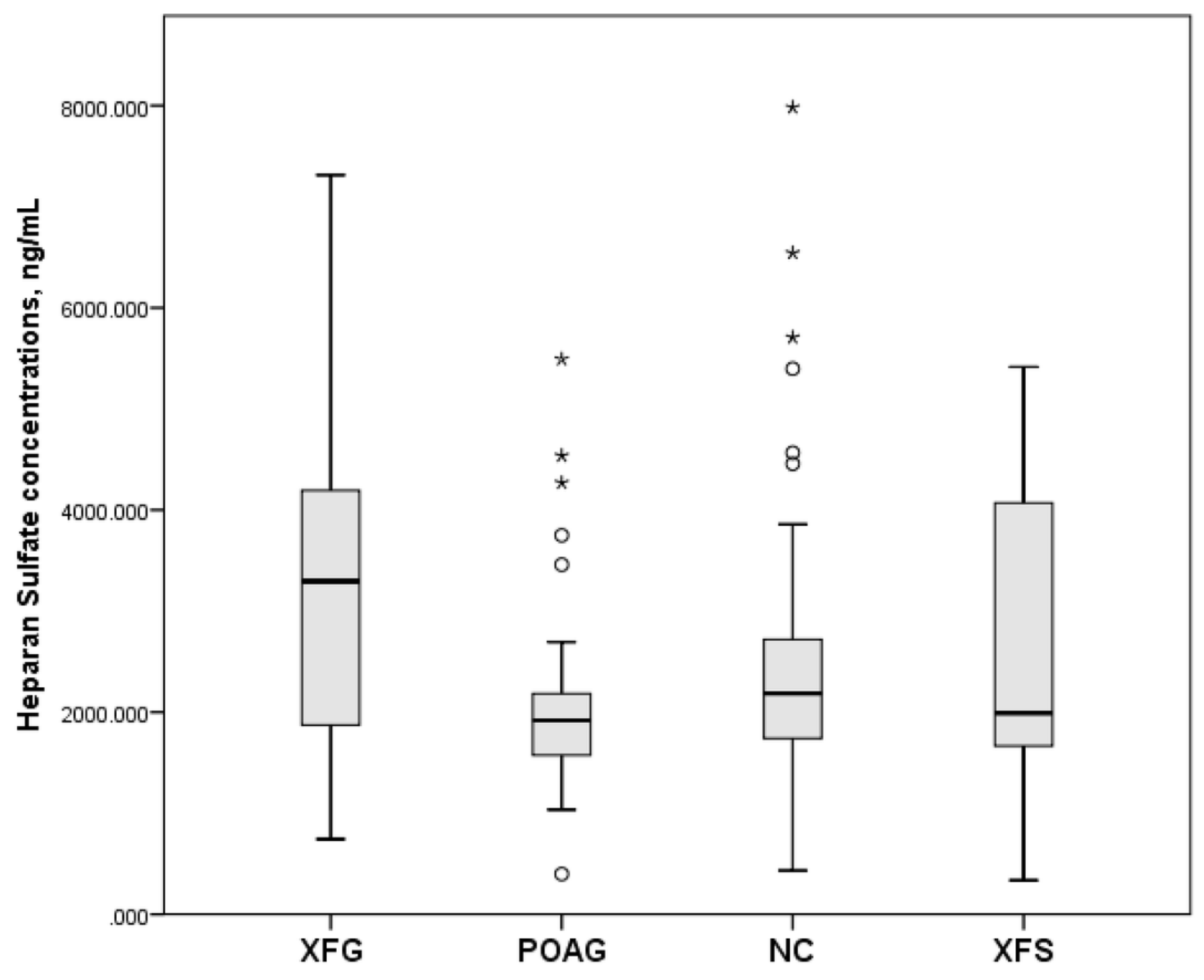


Figure 2

Serum Chondroitin Sulfate concentrations, $\mathrm{ng} / \mathrm{mL}$ in subjects with XFG, POAG, NC, XFS.

Box plots present median, bars are minimum and maximum values, circles are outliers and stars are extreme values.

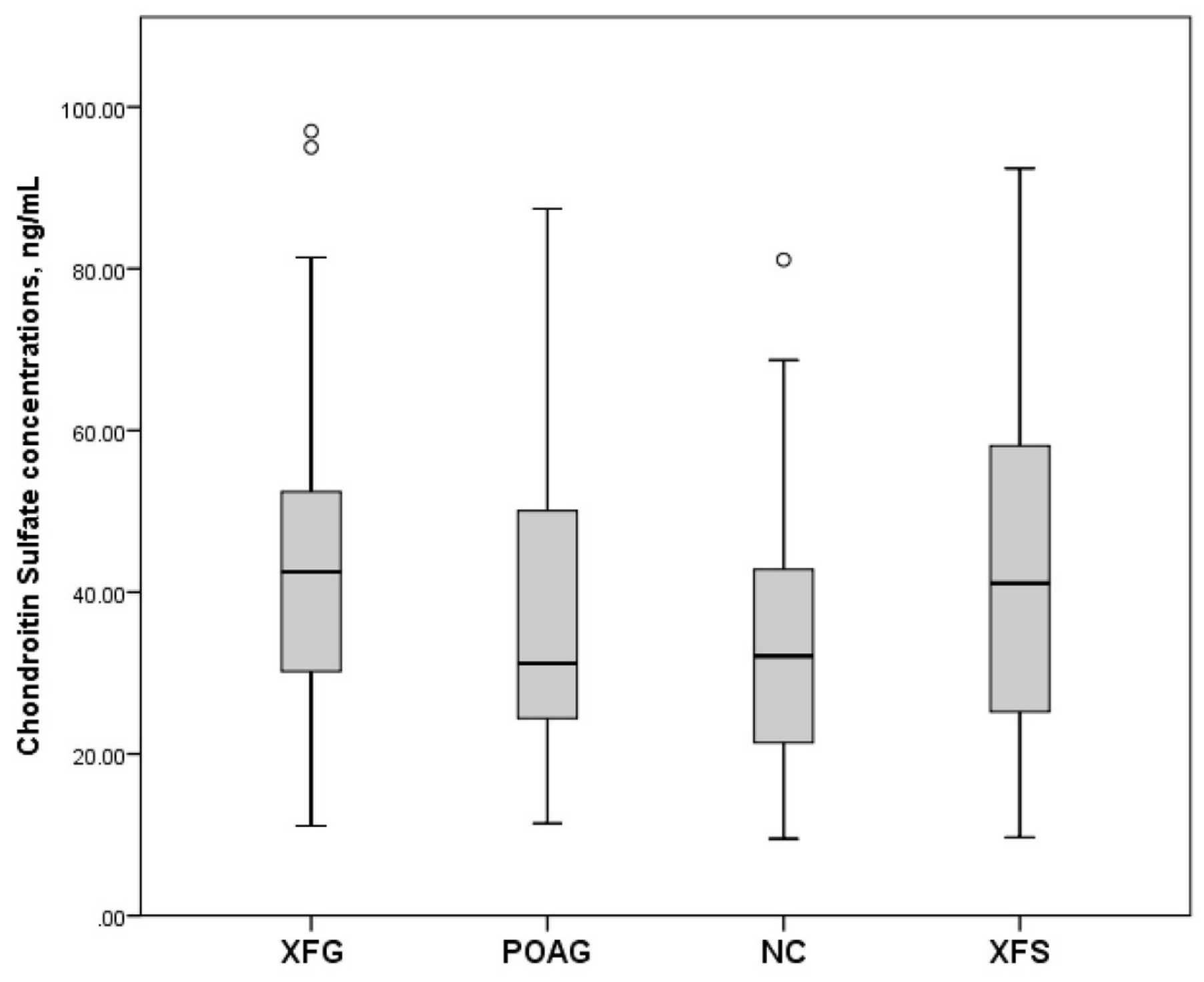


Figure 3

Serum Heparan Sulfate concentrations, $\mathrm{ng} / \mathrm{mL}$ in XFM and non XFM groups.

Box plots present median, bars are minimum and maximum values, circles are outliers and stars are extreme values. XFM group: XFG and XFS; Non XFM group: POAG and NC.

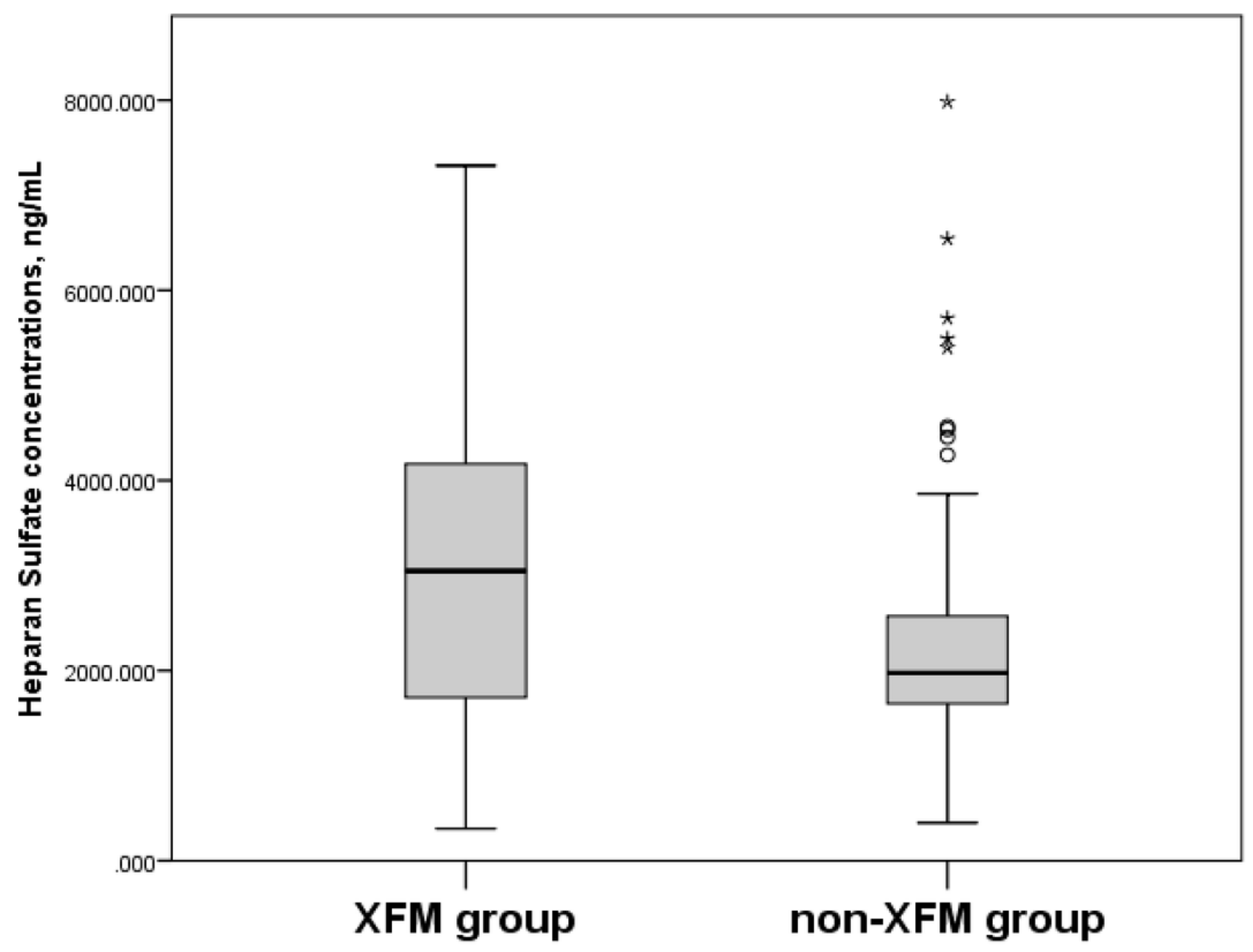


Figure 4

Serum Chondroitin Sulfate concentrations, $\mathrm{ng} / \mathrm{mL}$ in XFM and non XFM groups. Box plots present median, bars are minimum and maximum values, circles are outliers and stars are extreme values. XFM group: XFG and XFS; Non XFM group: POAG and NC.

Box plots present median, bars are minimum and maximum values, circles are outliers and stars are extreme values. XFM group: XFG and XFS; Non XFM group: POAG and NC.

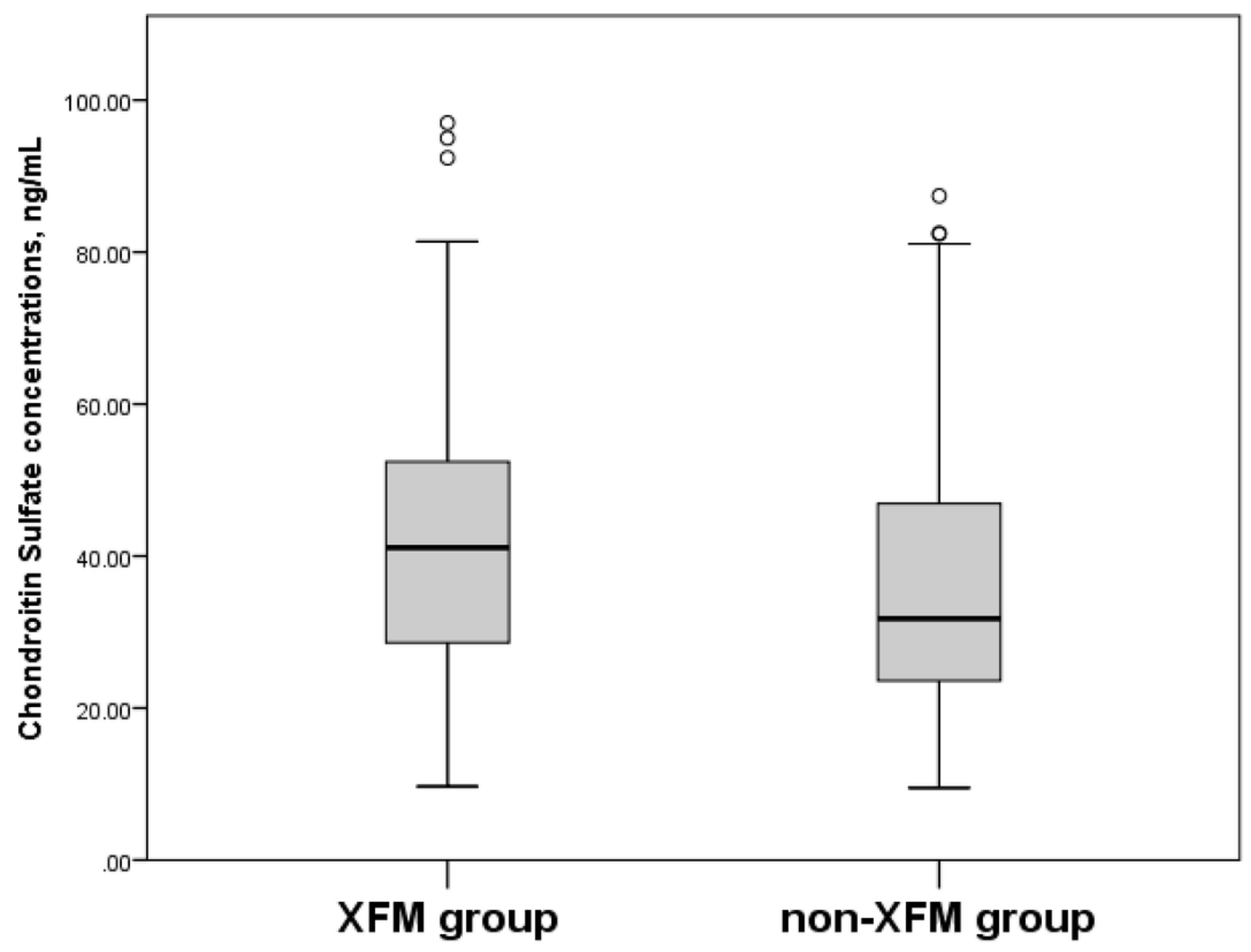


Figure 5

Area under the ROC curve of HS in patients with XFG compared to NC

ROC curve drawn for the comparison of serum heparan sulfate concentration between patients with exfoliative glaucoma and normal controls and area under the ROC curve was $0.62(95 \% \mathrm{Cl} 0.510-0.741), p=0.032$.

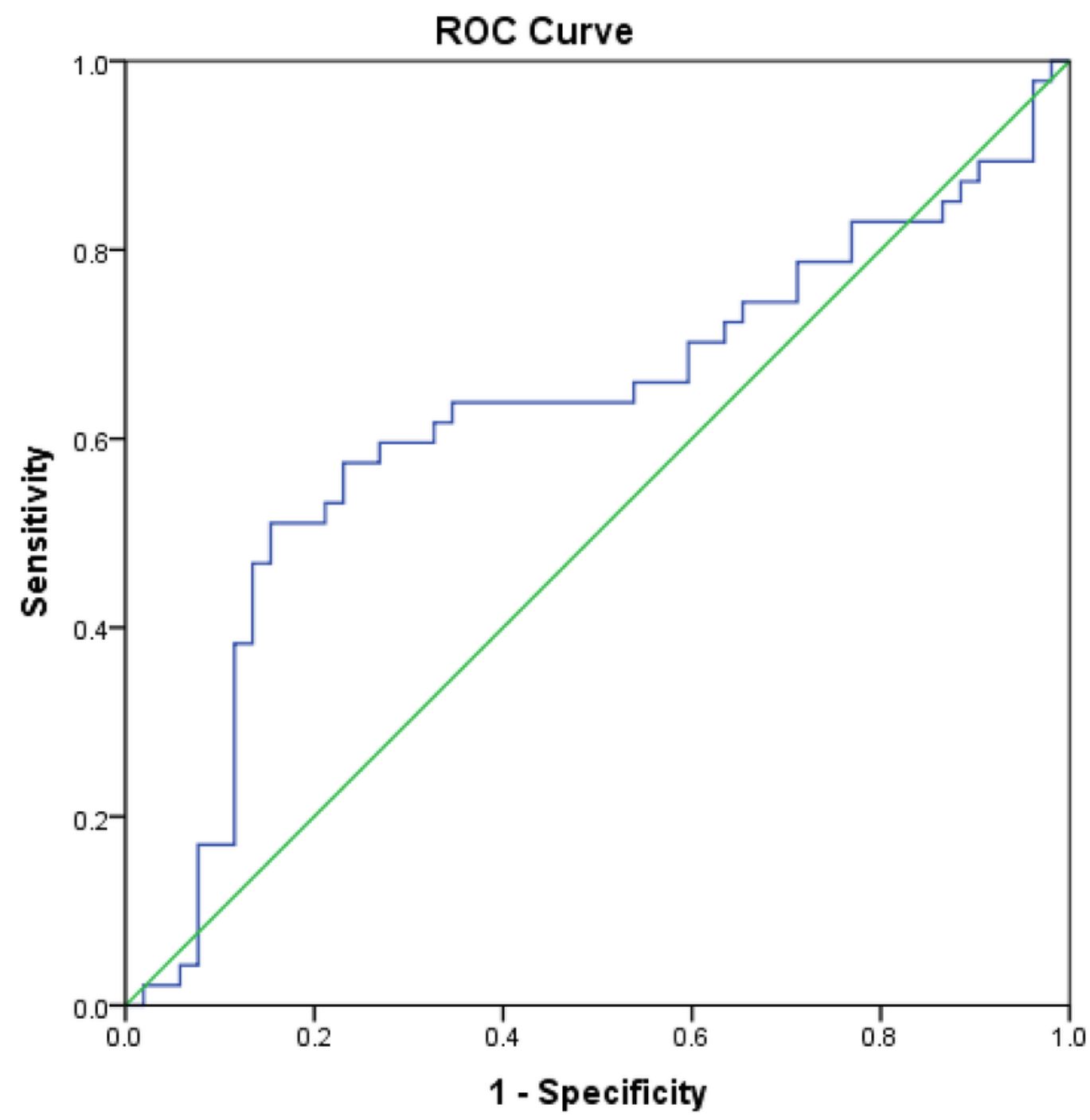


Figure 6

Area under the ROC curve of CS in patients with XFG compared to NC

ROC curve drawn for the comparison of serum chondroitin sulfate concentration between patients with exfoliative glaucoma and normal controls and area under the ROC curve was $0.62(95 \% \mathrm{Cl} 0.508-0.730), p=0.041$.

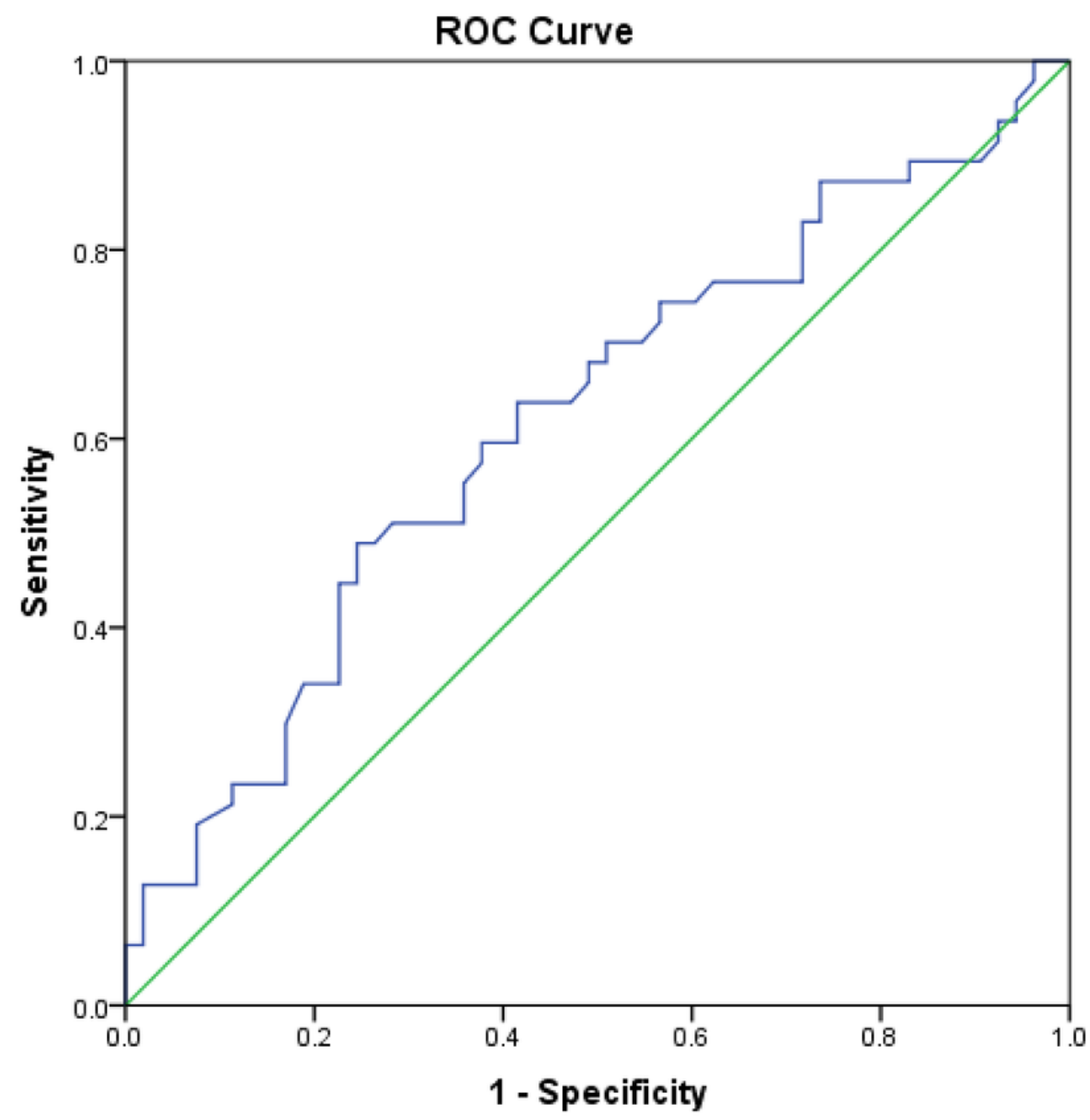

